\title{
Large-Eddy Simulation with Near-Wall Modeling Using Weakly Enforced No-Slip Boundary Conditions
}

\author{
Roozbeh Golshan ${ }^{1}$, Andrés E. Tejada-Martínez ${ }^{1, *}$, Mario Juha $^{1 *}$, Yuri Bazilevs ${ }^{2 *}$ \\ ${ }^{1}$ Department of Civil \& Environmental Engineering, University of South Florida, 4202 East \\ Fowler Avenue ENB 118, Tampa, FL 33620, USA \\ ${ }^{2}$ Department of Structural Engineering, University of California, San Diego, 9500 Gilman Drive \\ MC0085, La Jolla, CA 92093, USA
}

\begin{abstract}
In the present paper, weakly enforced no-slip wall boundary conditions are revisited in the context of Large-Eddy Simulations (LES) with near-wall modeling. A new formulation is proposed in the framework of weakly enforced no-slip conditions that is better aligned with traditional near-wall modeling approaches than its predecessors. The new formulation is tested on turbulent open-channel flows at friction-velocity-based Reynolds numbers $R e_{\tau}=395$ and 950 benchmark problems. The computations are performed using the Residual-Based Variational Multiscale (RBVMS) formulation of LES, discretized using Isogeometric Analysis (IGA) based on Non-Uniform Rational B-Splines (NURBS). The new near-wall model formulation gives more accurate results for the mean flow and
\end{abstract}

\footnotetext{
${ }^{*}$ Corresponding author; e-mail: aetejada@usf.edu; tel: 813-974-1738; fax: 813-974-2275
} 
velocity fluctuations than its older versions, while exhibiting better numerical stability than traditional near-wall modeling techniques.

Keywords: Near-wall modeling, weakly enforced no-slip conditions, boundary

layers, Isogeometric Analysis, open-channel flow, law-of-the-wall

\section{Introduction}

In large-eddy simulations (LES) [31] of wall-bounded turbulent flows, nearwall sharp gradients of the velocity field, and small eddies scaling with distance to the wall, give rise to undesired computational costs associated with fine grids and small time steps needed to resolve these features. Near-wall modeling is often employed to significantly reduce these costs [28]. In LES with near-wall modeling (LES-NWM), while the core flow is reasonably well resolved, the unresolved near-wall region is modeled through suitable boundary conditions, which obviates the need to use small time steps and fine meshes in the near-wall region. This is in contrast to LES with near-wall resolution (LES-NWR), in which the mesh is made finer near the wall, and the time-step size is reduced in order to capture the finer spatial and temporal scales of near-wall turbulence. With the significant reduction in computational cost brought about with near-wall modeling, more realistic problems in terms of computational-domain size, geometric complexity, and Reynolds number may be solved with LES-NWM as opposed to with LES- 
NWR or direct numerical simulation (DNS). The latter two are mostly employed to study turbulent-flow physics at relatively low Reynolds number in configurations of reduced geometric complexity.

In traditional near-wall modeling, instead of imposing the no-slip boundary conditions at the wall, the wall shear stress boundary conditions are prescribed. The following procedure is usually employed: Assuming the LES-resolved mean flow satisfies the log law, which is part of the well-known law-of-the-wall [32], the associated wall friction velocity is extracted, and, in turn, used to compute the magnitude of the prescribed wall shear stress. (The direction of the prescribed wall shear stress vector is given by the slip velocity.) Because the wall friction velocity is a nonlinear function of the LES-resolved flow velocity, an iterative procedure is necessary to compute it. We also note that the LES-resolved mean velocity used to extract the wall shear stress from the law-of-the-wall is often computed by averaging the flow field in space, typically over homogeneous flow directions, which makes the stress boundary conditions nonlocal.

An alternative approach to reduce mesh- and time-step-size requirements in wall-bounded turbulent flows was introduced in [8]. The approach is based on socalled weak enforcement of Dirichlet no-slip conditions at the solid wall. More specifically, the formulation at the discrete level is based on the variational (or 
weak) form of the Navier-Stokes equations, which is augmented by terms that enforce no-slip conditions weakly as Euler-Lagrange conditions. This construction results in a numerical technique that satisfies no-slip conditions exactly only in the limit as the mesh size at the wall approaches zero. As a result, for a given mesh, the method allows a certain amount of flow slip at the wall, which scales with the appropriate power of the wall-normal mesh size. Allowing the flow to slip at the solid boundary removes some of the burden from the boundary-layer mesh to resolve the sharp velocity gradients near the wall, which, in turn, results in remarkably accurate solutions for boundary-layer flows on meshes that would be considered "too coarse" by most CFD practitioners. (See references $[10,9,1,18,19]$ that highlight the coarse-mesh accuracy of weakly enforced no-slip conditions, both for well-known turbulent flow benchmark problems as well as for engineering applications.)

The method of weak enforcement of no-slip conditions is an extension of Nitsche's idea for imposing essential boundary conditions weakly [27], and is now commonly referred to as Nitsche's method. The method may also be thought of as the SIPG Discontinuous Galerkin technique (see [48]) applied only at the solid boundary. An important component of the weak boundary condition formulation is the penalty term whose integrand contains the deviation of the discrete 
solution from the no-slip condition at the wall. The integrand of the penalty term is also proportional to a mesh-dependent penalty parameter $\tau_{B}$ designed to ensure numerical stability and optimal convergence under mesh refinement. As such, weak imposition of no-slip conditions is based on numerical considerations rather than physical ones.

The link between weakly enforced no-slip conditions and near-wall modeling was first recognized and exploited in [10]. In the aforementioned reference the parameter $\tau_{B}$ was computed following the law-of-the-wall by considering the penalty term as being representative of the shear stress at the wall. While slight improvement in the results was observed relative to the purely numerical design of $\tau_{B}$, we recognize that the formulation in [10] was not completely consistent with the law-of-the-wall for the following reasons: 1 . Only the penalty term was representative of the shear stress at the wall, while the resolved molecular viscous shear stress was neglected; 2. The resolved LES velocity at the wall, instead of that inside the log layer, was employed to compute the wall shear stress and the penalty parameter $\tau_{B}$. This motivates the development of an improved formulation for weak enforcement of the non-slip boundary conditions, which we present in this paper. The merit of this new formulation is that it is designed to inherit the positive numerical attributes of the original formulations (i.e., stability and optimal 
convergence) while being consistent with the-law-of-the-wall. Furthermore, this better alignment with the law-of-the-wall opens the door for future improvements of the new formulation following the developments already made for traditional near-wall modeling [28].

The manuscript is organized as follows. Section 2 presents the Navier-Stokes equations of incompressible flows in the strong and weak forms and provides some details of the residual-based variational multiscale (RBVMS) formulation of LES [6, 2, 11]. Section 3 provides the details of a traditional near-wall model, as well as near-wall models based on weak imposition of no-slip conditions. The new near-wall model formulation is presented in Section 4. Sections 5 focuses on the numerical results. We compute pressure-gradient-driven turbulent openchannel flows at friction-velocity Reynolds numbers $R e_{\tau}=395$ and 950 using an isogeometric analysis (IGA) discretization based on Non-Uniform Rational BSplines (NURBS) $[22,15]$. A comparison of LES-NWM results for the traditional near-wall model, weak BC formulation from [10, 9], and its improved version proposed in the current work is performed. Conclusions are drawn in Section 6. 


\section{Navier-Stokes Equations, RBVMS Formulation, and Near-Wall Modeling}

Let $\Omega \in \mathbb{R}^{3}$ be the problem domain and let $\Gamma$ denote its boundary. A conservative form of the dimensionless Navier-Stokes equations of incompressible flows in the Eulerian frame may be written as

$$
\begin{array}{r}
\frac{\partial \boldsymbol{u}}{\partial t}+\nabla \cdot(\boldsymbol{u} \otimes \boldsymbol{u})+\nabla p-\nabla \cdot\left(2 \nu \nabla^{s} \boldsymbol{u}\right)=\boldsymbol{f} \text { in } \Omega \\
\nabla \cdot \boldsymbol{u}=0 \text { in } \Omega,
\end{array}
$$

where Eqs. (1) and (2) represent conservation of linear momentum and mass, respectively, assuming the fluid density is constant. In the above equations, $\boldsymbol{u}$ and $p$ are the fluid velocity and pressure (divided by density), $\nu$ is the kinematic viscosity, $\nabla^{s}=\frac{1}{2}\left(\nabla+(\nabla)^{T}\right)$ is the symmetric spatial gradient, and $\boldsymbol{f}$ is a body force per unit mass.

The RBVMS formulation of the Navier-Stokes equations of incompressible flows is employed in this work $[6,2,11]$. RBVMS originates from stabilized and multiscale methods for fluid mechanics [13, 47, 21, 23]. In [6] it was derived and presented for the first time in the context of subgrid-scale modeling for LES. In [5] a moving-domain extension of RBVMS was presented in the framework of an Arbitrary Lagrangian-Eulerian (ALE) formulation [20], and later called the ALE-VMS method [35]. The space-time version of RBVMS, called ST-VMS, 
was recently proposed in [41] and successfully employed in a number of fluid mechanics and fluid-structure interaction simulations in $[35,7,39,45,36]$. In [6] it was shown RBVMS performs well on laminar and turbulent flows, and discrete solutions converge rapidly to DNS while yielding LES-like solutions on intermediate meshes. For better approximation of thin boundary layers near no-slip walls various wall-modeling approaches may be used. Here we focus on classical wallfunction-based techniques as well as more recently proposed methods for weak enforcement of the no-slip Dirichlet boundary conditions [8].

The space-discrete formulation that combines RBVMS and near-wall modeling may be stated as: Find $\left\{\boldsymbol{u}^{h}, p^{h}\right\} \in \mathcal{V}^{h}$ such that $\forall\left\{\delta \boldsymbol{u}^{h}, \delta p^{h}\right\} \in \mathcal{W}^{h}$,

$$
\begin{aligned}
B\left(\left\{\delta \boldsymbol{u}^{h}, \delta p^{h}\right\},\left\{\boldsymbol{u}^{h}, p^{h}\right\}\right) & +B_{v m s}\left(\left\{\delta \boldsymbol{u}^{h}, \delta p^{h}\right\},\left\{\boldsymbol{u}^{h}, p^{h}\right\}\right) \\
& +B_{w m}\left(\left\{\delta \boldsymbol{u}^{h}, \delta p^{h}\right\},\left\{\boldsymbol{u}^{h}, p^{h}\right\}\right)=\left(\delta \boldsymbol{u}^{h}, \boldsymbol{f}\right)_{\Omega},
\end{aligned}
$$

where $\mathcal{V}^{h}$ denotes the discrete solution space for velocity-pressure pairs $\left\{\boldsymbol{u}^{h}, p^{h}\right\}$, $\mathcal{W}^{h}$ denotes the discrete space of linear-momentum and continuity-equation testfunction pairs $\left\{\delta \boldsymbol{u}^{h}, \delta p^{h}\right\}$, and $(\cdot, \cdot)_{A}$ denotes an $L_{2}$-inner product over the domain A.

The semi-linear forms in the formulation given by Eq. (3) are defined in what 
follows.

$$
\begin{array}{r}
B(\{\boldsymbol{w}, q\},\{\boldsymbol{u}, p\})=\left(\boldsymbol{w}, \frac{\partial \boldsymbol{u}}{\partial t}\right)_{\Omega}-(\nabla \boldsymbol{w}, \boldsymbol{u} \otimes \boldsymbol{u})_{\Omega}+(q, \nabla \cdot \boldsymbol{u})_{\Omega} \\
-(\nabla \cdot \boldsymbol{w}, p)_{\Omega}+\left(\nabla^{s} \boldsymbol{w}, 2 \nu \nabla^{s} \boldsymbol{u}\right)_{\Omega}
\end{array}
$$

is the Galerkin part of the weak form. Furthermore,

$$
\begin{array}{r}
B_{v m s}(\{\boldsymbol{w}, q\},\{\boldsymbol{u}, p\})=-\left(\nabla \boldsymbol{w}, \boldsymbol{u}^{\prime} \otimes \boldsymbol{u}+\boldsymbol{u} \otimes \boldsymbol{u}^{\prime}+\boldsymbol{u}^{\prime} \otimes \boldsymbol{u}^{\prime}\right)_{\Omega} \\
-\left(\nabla \cdot \boldsymbol{w}, p^{\prime}\right)_{\Omega}-\left(\nabla q, \boldsymbol{u}^{\prime}\right)_{\Omega} .
\end{array}
$$

are the RBVMS terms, where the pair $\left\{\boldsymbol{u}^{\prime}, p^{\prime}\right\}$ denotes the velocity and pressure subgrid scales (i.e., the scales that are too small to be reasonably approximated on a given mesh). As in [6], the subgrid scales are modeled as

$$
\begin{aligned}
& \boldsymbol{u}^{\prime}=-\tau_{M}\left(\frac{\partial \boldsymbol{u}}{\partial t}+\boldsymbol{u} \nabla \boldsymbol{u}+\nabla p-\nu \Delta \boldsymbol{u},-\boldsymbol{f}\right) \\
& p^{\prime}=-\tau_{C} \nabla \cdot \boldsymbol{u}
\end{aligned}
$$

where $\tau_{M}$ and $\tau_{C}$ are the subgrid-scale parameters defined later in the section. The subgrid-scale parameters are also known as stabilization parameters due to the similarities between RBVMS and stabilized and multiscale methods for fluid mechanics. Note that the fine scales are proportional to the residuals of the NavierStokes equations, which renders the numerical methodology consistent. Also note 
that the momentum-equation residual is in the advective form, which may be derived from the conservative form given by Eq. (1) using the incompressibility constraint from Eq. (2).

The parameter $\tau_{M}$ is taken as

$$
\tau_{M}=\left(\frac{4}{\Delta t^{2}}+\boldsymbol{u}^{h} \cdot \mathbf{G} \boldsymbol{u}^{h}+C_{I} \nu^{2} \mathbf{G}: \mathbf{G}\right)^{-\frac{1}{2}}
$$

where $\Delta t$ is time step and $C_{I}$ is a positive real constant, arising in error analysis (see, e.g., [25]), independent of the mesh size and problem material parameters. Furthermore, $\mathbf{G}=\left[G_{i j}\right]$, where

$$
G_{i j}=\frac{\partial \xi_{k}}{\partial x_{i}} \frac{\partial \xi_{k}}{\partial x_{j}}
$$

is the metric tensor of the mapping from the parametric to the physical domain of the element. The parameter $\tau_{C}$ is given by

$$
\tau_{C}=\frac{1}{\tau_{M} \operatorname{trG}}=\frac{1}{\tau_{M} G_{k k}} .
$$

Finally, the semi-linear form $B_{w m}$ in Eq. (3) gives the contribution of the near-wall modeling terms to the discrete formulation, and presents the main focus of this paper. The different choices considered for $B_{w m}$ are presented in the following sections. 


\section{Near-Wall Modeling and Weakly Enforced No-Slip Boundary Conditions}

In this section we present the formulation corresponding to a traditional nearwall model, and two formulations based on weakly enforced essential no-slip boundary conditions developed in earlier work $[8,10,9]$. In the following developments, for simplicity, we assume that the no-penetration boundary condition at the no-slip wall is enforced strongly. Extension to weakly enforced no-penetration boundary condition is straightforward.

\subsection{Traditional near-wall modeling}

In traditional near-wall modeling, Dirichlet boundary conditions at the no-slip wall are replaced with wall shear stress (i.e., Neumann) boundary conditions. In the context of variational formulations of the Navier-Stokes equations the nearwall term typically takes the following form:

$$
B_{w m}(\{\boldsymbol{w}, q\},\{\boldsymbol{u}, p\})=\left(\boldsymbol{w}, u^{* 2} \frac{\boldsymbol{u}_{t}}{\left\|\boldsymbol{u}_{t}\right\|}\right)_{\Gamma_{\text {wall }}},
$$

where $\Gamma_{\text {wall }}$ denotes the no-slip wall portion of the boundary $\Gamma,\|\cdot\|$ is the Eu-

clidean norm, and $u^{* 2}$ and $\boldsymbol{u}_{t} /\left\|\boldsymbol{u}_{t}\right\|$ are the magnitude and direction of the wall shear stress vector, respectively. Here, $\boldsymbol{u}_{t}$ is the flow velocity tangential to the wall, or the "slip velocity", given by

$$
\boldsymbol{u}_{t}=\boldsymbol{u}-(\boldsymbol{u} \cdot \boldsymbol{n}) \boldsymbol{n}
$$


at the wall, where $\boldsymbol{n}$ is the unit outward wall-normal vector. In traditional wall modeling, wall-friction velocity $u^{*}$ is obtained by assuming that the LES-computed mean flow speed parallel to the wall at a point $A$ within the log layer satisfies the classical log law [29]:

$$
U^{A+}=\frac{U^{A}}{u^{*}}=\frac{1}{\kappa} \log \left(\frac{y^{A} u^{*}}{\nu}\right)+B,
$$

where $y^{A}$ denotes the wall-normal distance from the wall to point $A$, and $U^{A}$ denotes the mean LES-computed flow speed parallel to the wall at point $A$, namely,

$$
U^{A}=\left\langle\left\|\boldsymbol{u}_{t}^{A}\right\|\right\rangle
$$

Here, $\boldsymbol{u}_{t}^{A}$ is the LES-computed fluid velocity vector parallel to the wall at A, and the brackets denote averaging. Averaging is typically performed over homogeneous directions in the flow. We note that averaging, which is often required in order to obtain stable numerical results, renders the above near-wall model nonlocal. If there are no homogeneous directions in the flow, which is almost always the case for complex-geometry surfaces, some other form of averaging may be required for numerical stability.

In Eq. (12) the remaining coefficients are $\kappa=0.41$ (the von Karman constant) and $B=5.5$ (see [32]). Finally, Eq. (12) is nonlinear in $u^{*}$ and is thus solved iteratively for $u^{*}$ yielding the imposed traction magnitude $u^{* 2}$ (see Eq. (10)). 


\subsection{Near-wall modeling based on weak imposition of no-slip conditions}

Rather than prescribing the wall shear stress as in the case of traditional wall modeling, a different approach was introduced in [8] based on weak imposition of no-slip conditions at the wall. In this case the term $B_{w m}$ in Eq. (3) is defined as:

$$
\begin{array}{r}
B_{\text {wm }}(\{\boldsymbol{w}, q\},\{\boldsymbol{u}, p\})=\left(\boldsymbol{w},-2 \nu \nabla^{s} \boldsymbol{u} \cdot \boldsymbol{n}\right)_{\Gamma_{\text {wall }}} \\
+\left(-2 \nu \nabla^{s} \boldsymbol{w} \cdot \boldsymbol{n},(\boldsymbol{u}-\boldsymbol{g})\right)_{\Gamma_{\text {wall }}} \\
+\left(\boldsymbol{w}, \tau_{B}(\boldsymbol{u}-\boldsymbol{g})\right)_{\Gamma_{\text {wall }}}
\end{array}
$$

which sets $\boldsymbol{u}=\boldsymbol{g}$, (in this work, $\boldsymbol{g}=\mathbf{0}$ ), at the wall, in the weak sense, or in the sense of Euler-Lagrange conditions. Description of the terms in Eq. (14), as given originally in [8], follows. The first term on the right side of Eq. (14) is referred to as the consistency term given than the Euler-Lagrange equations for Eq. (3) contain a term (arising from integration by parts) that cancels the consistency term. The second term on the right side of Eq. (14) is referred to as the adjoint consistency term. If the exact solution of the adjoint problem is used in Eq. (3) as the test function, then Eq. (3) is satisfied exactly. Adjoint consistency is linked to optimal convergence in lower-order norms [3]. The third term on the right side of Eq. (14) is the so-called penalty term, as it penalizes the deviation of the discrete solution from its prescribed value at the wall. In what follows, we show 
two definitions of the penalty parameter $\tau_{B}$, which emanate from considerations of numerical stability and optimal convergence under mesh refinement, as well as partial alignment with the law-of-the-wall.

As in the original reference [8], the penalty parameter $\tau_{B}$ in Eq. (14) may be chosen as

$$
\tau_{B}=\frac{C_{b} \nu}{h_{n}}
$$

where $h_{n}$ is the wall-normal element size given by

$$
h_{n}=2\left(\boldsymbol{n}^{T} \mathbf{G} \boldsymbol{n}\right)^{-\frac{1}{2}}
$$

and $C_{b}$ is an element-wise positive constant, which arises in error analysis, and which is independent of the mesh size and problem material parameters. The constant $C_{b}$ needs to be sufficiently large to guarantee numerical stability of the weak-boundary-condition formulation.

The weak imposition of the no-slip condition was re-visited in [10], and a modification was introduced with the goal of aligning the formulation with traditional wall modeling. For this, the weak-boundary-condition framework given by Eq. (14) was retained, however, rather than calculating $\tau_{B}$ as in Eq. (15), a new expression for $\tau_{B}$ was postulated by comparing the right side of Eq. (10) with the 
third term (i.e., the penalty term) in the right side of Eq. (14), resulting in

$$
\tau_{B}=\frac{u_{m o d}^{* 2}}{\left\|\boldsymbol{u}_{t}\right\|}
$$

Modified wall friction velocity $u_{m o d}^{*}$ is computed iteratively through Spalding's parameterization of the turbulent boundary layer [32], namely,

$$
y^{+}=f\left(u^{+}\right)=u^{+}+\mathrm{e}^{-\kappa B}\left(\mathrm{e}^{\kappa u^{+}}-1-\kappa u^{+}-\frac{\left(\kappa u^{+}\right)^{2}}{2}-\frac{\left(\kappa u^{+}\right)^{3}}{6}\right)
$$

with

$$
u^{+}=\frac{\left\|\boldsymbol{u}_{\text {slip }}\right\|}{u_{\text {mod }}^{*}}, \quad y^{+}=\frac{y^{A} u_{m o d}^{*}}{\nu}, \quad \text { and } \quad y^{A}=\frac{h_{n}}{C_{b}}
$$

Note that in this formulation the Spalding's formula is evaluated using $y^{A}=$ $h_{n} / C_{b}$ with the wall-slip speed $\left\|\boldsymbol{u}_{t}\right\|$ instead of the mean flow speed parallel to the wall at $y^{A}=h_{b} / C_{b}$, i.e., $\left\|\boldsymbol{u}_{t}^{A}\right\|$. Consequently, the weak boundary condition formulation makes use of a modified friction velocity $u_{m o d}^{*}$ instead of the "true" friction velocity, $u^{*}$.

As noted in [10], in the limit of wall-normal mesh refinement in the near-wall region (i.e., letting $h_{n}$ go to zero), $\left\|\boldsymbol{u}_{t}\right\| \rightarrow\left\|\boldsymbol{u}_{t}^{A}\right\|$, Spalding's formula given by Eq. (18) becomes $y^{+}=u^{+}$, and the penalty parameter $\tau_{B}$ in Eq. (17) becomes independent of $\left\|\boldsymbol{u}_{t}\right\|$ taking on the expression coincident with the earlier, purely 
numerical definition,

$$
\tau_{B}=\frac{\nu C_{b}}{h_{n}} .
$$

Thus, the seemingly disparate evaluation of Spalding's formula at $y_{A}=h_{n} / C_{b}$ with the wall-slip speed $\left\|\boldsymbol{u}_{t}\right\|$ (noted above) is a mathematical construct designed to yield the original formulation of the weak Dirichlet (no-slip) conditions in Eqs. (14) and (15) in the limit $h_{n} \rightarrow 0$. In this limit, the formulation for $\tau_{B}$ in Eq. (17) inherits the stability and accuracy attributes of the original formulation described in [8].

It is also important to note that, since in the Spalding's parameterization of the turbulent boundary layer $y^{+} \geq u^{+}$, it can be shown that $\tau_{B}$, given by Eq. (17) with Spalding's formula evaluated using $y_{A}=h_{n} / C_{b}$ and $\left\|\boldsymbol{u}_{t}\right\|$, satisfies the following lower bound:

$$
\tau_{B}=\frac{u_{m o d}^{* 2}}{\left\|\boldsymbol{u}_{t}\right\|} \geq \frac{\nu C_{b}}{h_{n}}
$$

In other words, the choice of $\tau_{B}$ given by Eq. (17) renders the weak boundary condition formulation stable for the entire parameterization of the turbulent boundary layer, from the log layer to the viscous sublayer. 


\section{Weakly-Enforced No-Slip Conditions Aligned with the Law-of-the-Wall}

The modified weak no-slip boundary condition formulation presented in the previous section is consistent with the law-of-the-wall for small $h_{n}$. However, when $h_{n}$ is large, it deviates from the law-of-the-wall, in part due to the disparate evaluation of the Spalding's formula described in the previous section. In this section, still within the framework of weakly-enforced no-slip conditions, a new definition of the penalty parameter $\tau_{B}$ is proposed such that the resulting formulation is fully aligned with the law-of-the-wall when $h_{n}$ is large.

Inserting the weak boundary condition terms in Eq. (14) into Eq. (3), it becomes apparent that the consistency and penalty terms in Eq. (14) combined act as the shear stress at the wall. The consistency term may be thought of as the resolved molecular shear stress, while the penalty term may be interpreted as providing the necessary additional shear stress (i.e., "penalty stress" or "subgrid shear stress") such that the sum of the two gives rise to the expected wall shear stress. This condition may be expressed as

$$
-2 \nu \nabla^{s} \boldsymbol{u} \cdot \boldsymbol{n}+\tau_{B}(\boldsymbol{u}-\boldsymbol{g})=u^{* 2} \frac{\boldsymbol{u}_{t}}{\left\|\boldsymbol{u}_{t}\right\|},
$$

which holds on $\Gamma_{\text {wall }}$ with $\boldsymbol{g}=\mathbf{0}$. Equation (22) may now be used to obtain the expression for $\tau_{B}$, by dotting the left and right sides of the equation with the unit 
vector $\boldsymbol{u}_{t} /\left\|\boldsymbol{u}_{t}\right\|$, and solving for the former as:

$$
\tau_{B}=\frac{1}{\left\|\boldsymbol{u}_{t}\right\|}\left[u^{* 2}-\nu\left(\frac{\partial\left\|\boldsymbol{u}_{t}\right\|}{\partial n}\right)_{\Gamma_{\text {wall }}}\right],
$$

where $\partial\left\|\boldsymbol{u}_{t}\right\| / \partial n$ denotes the wall-normal derivative of the flow velocity parallel to the wall.

The definition of $\tau_{B}$ via Eqs. (22) and (23) is inspired by a popular technique in RANS (Reynolds-Averaged Navier-Stokes) simulations (see, e.g., [12]) in which the underprediction of the wall shear stress by the resolved molecular viscous shear stress is offset by the introduction of an effective wall viscosity $\nu_{e}$ calculated from

$$
\nu_{e}\left(\frac{\partial\left\|\boldsymbol{u}_{t}\right\|}{\partial n}\right)_{\Gamma_{\text {wall }}}=u^{* 2}
$$

with the wall-normal derivative of $\left\|\boldsymbol{u}_{t}\right\|$ at the wall approximated as

$$
\left(\frac{\partial\left\|\boldsymbol{u}_{t}\right\|}{\partial n}\right)_{\Gamma_{\text {wall }}} \approx \frac{\left\|\boldsymbol{u}_{t}^{A}\right\|}{y^{A}}
$$

where $y^{A}$ is now the true normal distance from the wall to point A within the log layer. The approximation of $\frac{\partial\left\|\boldsymbol{u}_{t}\right\|}{\partial n}$ at the wall given by Eq. (25) is also employed in this work to evaluate $\tau_{B}$ using Eq. (23).

The above formulation, while aligned with the law-of-the-wall, does not recover the original definition for $\tau_{B}$ (i.e., $\tau_{B}=C_{b} \nu / h_{n}$ ) in the limit $h_{n} \rightarrow 0$. Instead, in this limit, $\tau_{B} \rightarrow 0$ because the resolved viscous shear stress approaches 
the total stress, which makes the right side of Eq. (23) approach zero. This behavior is not desired, since the method will not be stable in this limit. In order to avoid this instability and to recover the positive accuracy attributes of the original weakboundary-condition formulation, we propose to adopt the following definition of $\tau_{B}:$

$$
\tau_{B}=\max \left(\frac{1}{\left\|\boldsymbol{u}_{t}\right\|}\left[u^{* 2}-\nu\left(\frac{\partial\left\|\boldsymbol{u}_{t}\right\|}{\partial n}\right)_{\Gamma_{\text {wall }}}\right], \frac{\nu C_{b}}{h_{n}}\right) .
$$

That is, $\tau_{B}$ is computed according to Eqs. (23) and (15), the new and original definitions, respectively, and the maximum is taken as its final value. In this construction, in the case of relatively large $h_{n}, \tau_{B}$ is governed by Eq. (23). As the mesh is refined near the wall to better capture the viscous shear stress, $\tau_{B}$ eventually reverts to the definition given by Eq. (15). The former renders the formulation consistent with the law-of-the-wall in the regime of unresolved viscous shear stresses, and the latter gives the required stability and accuracy properties in the regime of well-resolved viscous shear stresses. In that sense, on a given mesh, the proposed formulation automatically adjusts to the flow regime in which it is deployed. 


\section{Numerical Example: Turbulent Open Channel Flow}

In this section we present computational results for the pressure-gradientdriven open channel flow at friction-velocity-based Reynolds numbers $R e_{\tau}=395$ and 950. We assess the performance of the methods presented, and also compare with the DNS results of [26] and [17].

\subsection{Problem setup}

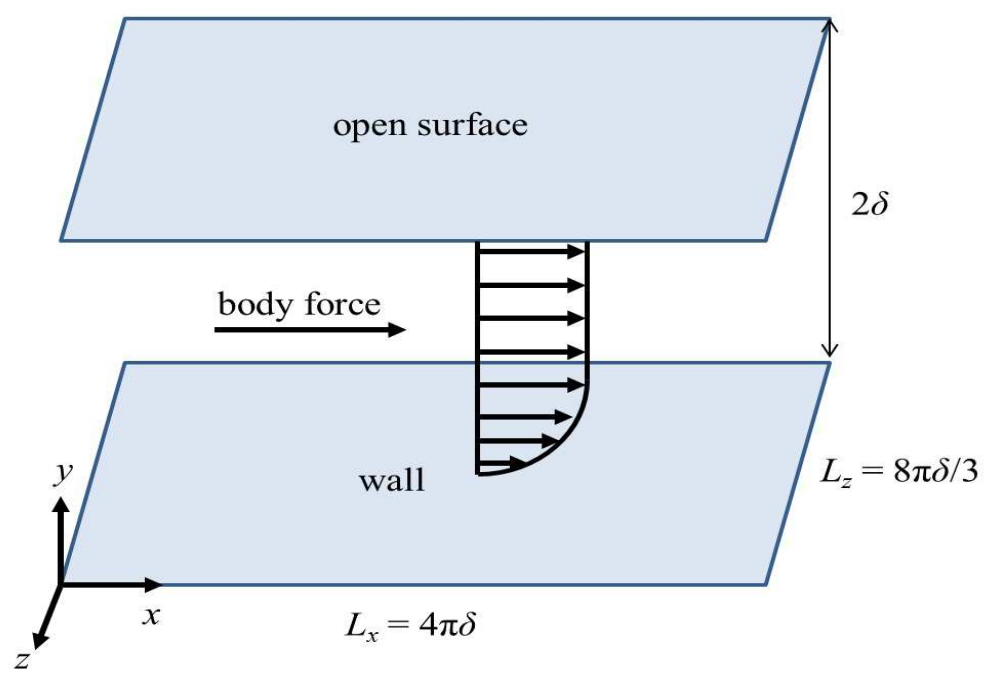

Figure 1: Computational domain.

The computational domain, depicted in Figure 1, is a rectangular channel with dimensions $4 \pi \delta \times 2 \delta \times \frac{8}{3} \pi \delta$ in the stream-wise $\left(x_{1}\right.$ or $x$ ), wall-normal ( $x_{2}$ or $y$ ), and span-wise $\left(x_{3}\right.$ or $z$ ) directions, respectively. The half-height of the domain (in 
the $y$-direction) is $\delta$. The open top surface of the channel is located at $y=2 \delta$ and the bottom wall at $y=0$.

The flow is driven by an imposed pressure gradient (or body force) in the $x$ direction and thus $\boldsymbol{f}=\left(f_{1}, 0,0\right)^{T}$ in Eq. (1). The first entry of this vector, $f_{1}$, is chosen such that $R e_{\tau}=395$ for the lower Reynolds number channel and $R e_{\tau}=950$ for the higher Reynolds number case. Here $R e_{\tau}=u^{*} \delta / \nu$ is the friction-velocity-based Reynolds number, where $u^{*}$ is the friction velocity and $\delta$ is the channel half-height. No-penetration boundary condition $(v=0)$ is imposed strongly at the open top surface and the bottom wall. Furthermore, zero shear stress is imposed at the top surface while the bottom wall is treated with the wall models described in the previous sections. Periodic boundary conditions are set in the flow homogeneous directions, that is, the stream-wise and span-wise directions.

Isogeometric analysis (IGA) is employed in the problem spatial discretization. Quadratic Non-Uniform Rational B-Splines (NURBS) that are $C^{1}$-continuous across mesh knots are employed in the computations. For the channel at $R e_{\tau}=395$, the mesh consists of $64 \times 34 \times 64$ quadratic NURBS elements in the $x, y$, and $z$ directions, respectively. For the channel at $R e_{\tau}=950$, the mesh consists of $64 \times 66 \times 64$ quadratic NURBS elements. The elements have uniform size in all directions, in- 
cluding the wall-normal direction. Details of simulations are presented in Table 1.

\begin{tabular}{lccccccc}
\hline & $L_{x}$ & $L_{y}$ & $L_{z}$ & $N_{x}$ & $N_{y}$ & $N_{z}$ & $\Delta t u^{*} / \delta$ \\
\hline \hline$R e_{\tau}=395$ & $4 \pi \delta$ & $2 \delta$ & $\frac{8}{3} \pi \delta$ & 64 & 34 & 64 & 0.00145 \\
$R e_{\tau}=950$ & $4 \pi \delta$ & $2 \delta$ & $\frac{8}{3} \pi \delta$ & 64 & 66 & 64 & 0.000641 \\
\hline
\end{tabular}

Table 1: Summary of domain sizes and mesh resolutions used in computations. $L_{x, y, z}$ is the domain length in the stream-wise, wall-normal, and span-wise direction, and $N_{x, y, z}$ is the number of basis functions in the stream-wise, wall-normal, and span-wise directions, respectively.

Remark In general, for NURBS of order $p$ and maximal continuity $p-1$, the number of basis functions in each tensor-product direction equals to $n+p$, where $n$ is the number of elements in this direction. When periodic boundary conditions are employed, the number of basis functions is reduced to $n$, which is independent of the polynomial order. This is in contrast to more standard $C^{0}$-continuous finite elements of order $p$, where the number of basis functions in a given tensor product direction is $p \cdot n+1$ (or $p \cdot n$ in the periodic case). This amounts to significant savings in the number of degrees of freedom for NURBS elements with respect to finite elements of the same order, especially in 3D. The interested reader is directed to $[22,15]$ for background on IGA and to $[6,2,9,4,46]$ for applications of 
IGA to turbulent flows.

The semi-discrete equations resulting from an IGA discretization are integrated in time using a fully-implicit second-order Generalized- $\alpha$ method [14, 24], and the underlying nonlinear equation systems are solved using inexact NewtonKrylov approach (see [6] for details). The dimensionless time-step size in the simulations is $\Delta t u^{*} / \delta=0.00145$ for the $R e_{\tau}=395$ flow and $\Delta t u^{*} / \delta=0.000641$ for the $R e_{\tau}=950$ case. Each simulation ran over a sufficiently long enough time period to obtain converged mean-flow and velocity-fluctuation statistics.

Some of the methods presented in this paper require the evaluation of the resolved LES velocity parallel to the wall at a chosen location within the log layer. In the results presented here, point $y^{A}$ (see Eq. (12)) is taken at the first mesh knot off the wall, which corresponds to $y^{+}=24.7$ for the channel flow at $R e_{\tau}=395$ and $y^{+}=29.7$ for the channel flow at $R e_{\tau}=950$.

In the LES of the channel flows described earlier, the following methods are implemented and compared: a) The traditional wall model presented in Section 3.1, labeled TWM in the figures; b) The weakly enforced essential-boundarycondition formulation presented in Section 3.2 with $\tau_{B}$ defined in Eq. (17), labeled WD2 in the figures; c) The new weakly enforced essential-boundary-condition formulation with $\tau_{B}$ defined in Eq. (26), labeled WD3 in the figures. The results 
using the weakly enforced essential-boundary-condition formulation with $\tau_{B}$ defined in Eq. (15) (or better yet WD1) are not presented since these are nearly indistinguishable from the WD2 case. This is consistent with the findings in [10]. Thus, improvements in simulation results yielded by the WD3 formulation over WD2 to be described below also apply to improvements yielded by WD3 over WD1.

Remark It is important to note that in the case of TWM implementation, the resolved LES velocity at a point $y^{A}$ used to evaluate the wall shear stress is obtained by averaging this quantity over the homogeneous flow directions. Without such averaging the TWM formulation looses stability. This is in contrast to the formulations based on the framework of weakly enforced boundary conditions, where no averaging is necessary for stability, and so it is not performed. This is an important point to consider when applying wall-function techniques in practical problems where there are no homogeneous flow directions over which one can average the flow field. 


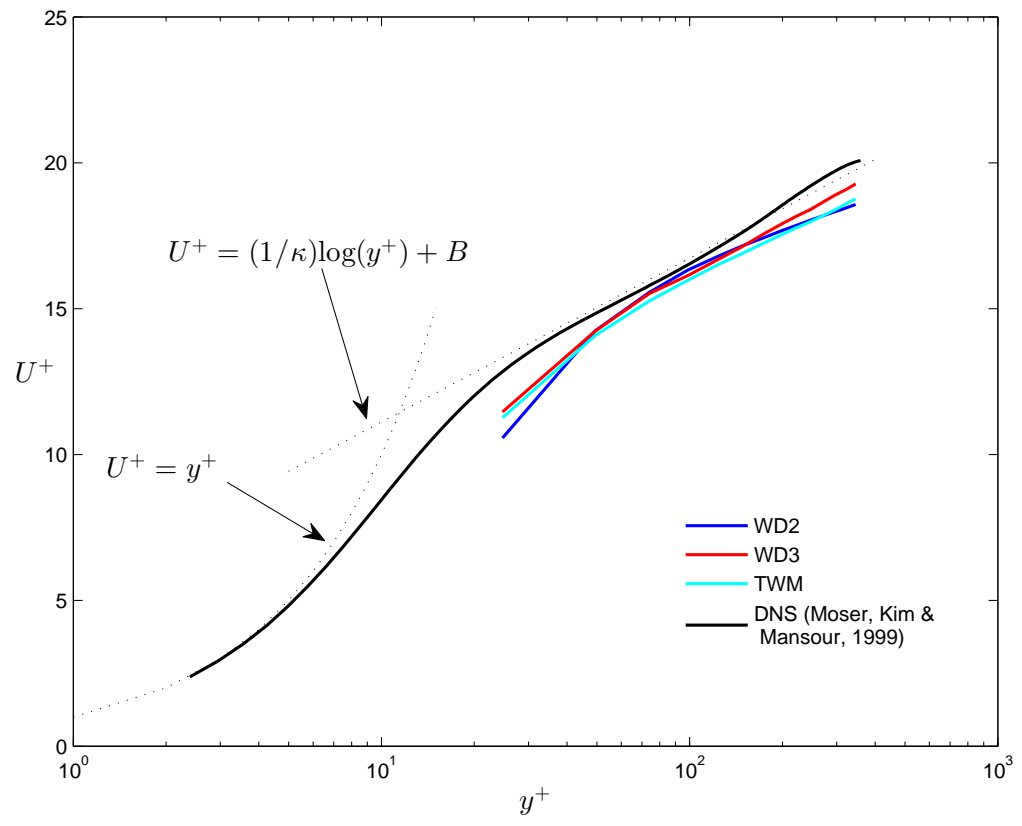

Figure 2: Mean velocity in log wall units from the near-wall model computations of turbulent channel flow at $R e_{\tau}=395$. DNS results from [26] and the theoretical log law with $\kappa=0.41$ and $B=5.5$ are also plotted for comparison.

\subsection{Statistics for Turbulent Open Channel Flow at $\operatorname{Re}_{\tau}=395$}

Figure 2 shows the mean velocity in log wall units from the LES with nearwall model computations for the $R e_{\tau}=395$ channel. Comparison with the DNS data from [26] is also presented. The mean velocity profiles obtained are in overall good agreement with the DNS and the theoretical log law. For $y^{+}>50$, the TWM gives rise to an under-prediction of the mean velocity relative to the vari- 
ous near-wall treatments based on weak imposition of the no-slip condition. The newly introduced WD3 leads to a slightly improved prediction of mean velocity compared to its predecessor WD2, as well as TWM.

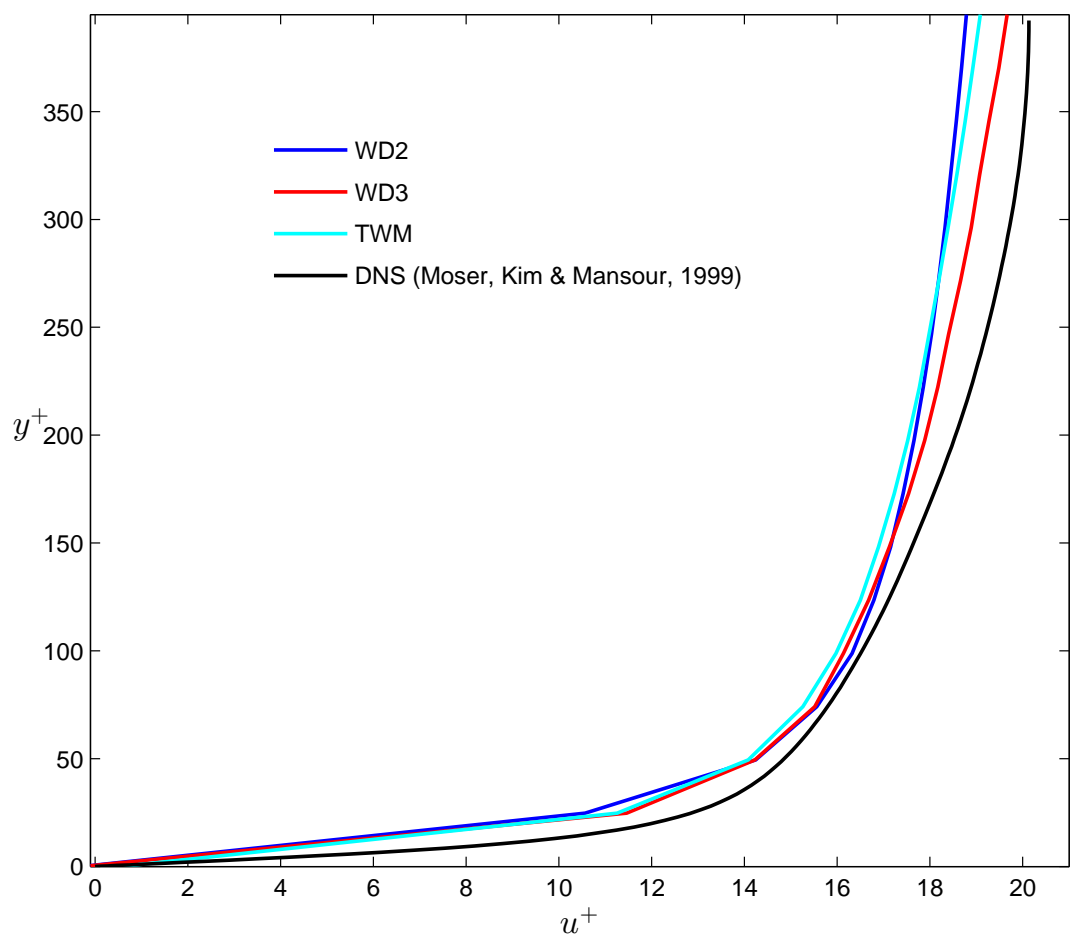

Figure 3: Mean velocity in wall units from the near-wall model computations of turbulent channel flow at $R e_{\tau}=395$. DNS results are also plotted for comparison.

Looking at the mean velocity plots for the $R e_{\tau}=395$ channel shown in Figure 3, it can be seen that WD3 is closest to the DNS in predicting the bulk velocity, as compared to both WD2 and TWM. 
Figure 4 shows root-mean-square (rms) of the velocity in wall units for the flow at $R e_{\tau}=395$. The WD3 formulation results in an improved prediction of the stream-wise velocity rms $\left(u_{r m s}\right)$ compared with WD2 in the range $100<$ $y^{+}<225$. The WD3 formulation gives $u_{r m s}$ that is in excellent agreement with the DNS data within this range. The WD3 formulation also provides an improved prediction of the peak $u_{r m s}$. Furthermore, the WD3 formulation leads to significant improvements over WD2 in terms of span-wise $\left(w_{r m s}\right)$ and wall-normal $\left(w_{r m s}\right)$ velocity rms through most of the channel. Overall, the velocity rms prediction with the WD3 formulation is in fairly close agreement with that obtained with the TWM formulation.

Instantaneous snapshots of flow speed for different near-wall treatments for the $R e_{\tau}=395$ case are shown in Figure 5. The TWM and WD3 wall treatments lead to a more "active" near-wall region characterized by more energetic eddies emanating from this region compared to WD2. In the WD2 formulation the turbulent features near the wall appear somewhat more suppressed, which, in turn, leads to underprediction of the velocity fluctuations relative to WD3 and TWM formulations and the DNS (see Figure 4). 


\subsection{Statistics for Turbulent Open Channel Flow at $R e_{\tau}=950$}

Figures 6 and 7 show the mean velocity from the LES with near-wall modeling for the $R e_{\tau}=950$ flow. Comparison with the DNS data from [16] is also presented. The newly introduced WD3 leads to improved prediction of mean velocity compared to its predecessor WD2, but the prediction is not as accurate as the prediction with TWM for this Reynolds number. Although TWM leads to an accurate velocity, especially within the log-layer, it leads to an over-prediction of the near-wall turbulence intensities, as was also seen earlier for the $R e_{\tau}=395$ flow.

Figure 8 shows rms of the velocity in wall units. Overall, the stream-wise and span-wise velocity rms prediction with the WD3 formulation is in fairly close agreement with that obtained with the TWM for $y^{+}>600$. Unlike TWM which leads to high stream-wise velocity rms near the wall, both WD3 and WD2 are in closer agreement with the DNS in the range $y^{+}<100$.

For the $R e_{\tau}=950$ flow, TWM leads to an over-prediction in the peak value of stream-wise velocity rms with respect to the DNS (Figure 8). Meanwhile WD2 leads to an accurate prediction of the peak. In the $R e_{\tau}=395$ flow (Figure 4), peak stream-wise velocity rms predictions by TWM and WD2 are seen to be shifted down (with respect to the corresponding DNS) compared to their predictions for 
the $R e_{\tau}=950$ flow. Thus, in the flow at $R e_{\tau}=395$, TWM leads to an improved prediction of the peak compared to the prediction at $R e_{\tau}=950$. Furthermore, in the $R e_{\tau}=395$ flow, WD2 leads to an under-prediction of the peak and thus a worse prediction compared to the prediction at $R e_{\tau}=950$. This behavior of WD2 and TWM may be attributed to the finer resolution of the mesh in the horizontal directions ( $x$ and $z$ ) for the $R e_{\tau}=395$ flow compared to the $R e_{\tau}=950$ case (see Table 1), causing the predicted peak of stream-wise velocity rms to shift down with improved resolution. The behavior of peak stream-wise velocity rms in the LES with WD3 is markedly different, as the predicted value is not greatly affected by the mesh resolution. That is, WD3 leads to good predictions of the peak in both flows, $R e_{\tau}=395$ and $R e_{\tau}=950$, despite the different mesh resolutions in these cases.

Instantaneous snapshots of flow speed for different near-wall treatments are shown in Figure 9 for the flow at $R e_{\tau}=950$. Unlike in the $R e_{\tau}=395$ case, for which WD2 led to significantly more damped near-wall flow speed fluctuations, in the $R e_{\tau}=950$ flow, the WD2 and WD3 formulations lead to fluctuations of similar intensities, both being less intense than the fluctuations obtained with TWM. This is consistent with the velocity rms profiles described earlier. 


\subsection{Simulation statistics}

We conclude the section by presenting some simulation statistics for the three near-wall modeling formulations considered in this work. As mentioned earlier, the underlying nonlinear equation systems are solved to a prescribed tolerance using the inexact Newton-Krylov approach. The linear equation systems are solved to a prescribed tolerance using a Generalized Minimal Residual (GMRES) technique [30] with diagonal preconditioning. In all cases, the relative tolerance of the

nonlinear equation system is set to $10^{-6}$ for both the momentum and continuity equation residuals. (This is a much lower threshold than is typically employed in more practical simulations.) The maximum number of nonlinear iterations per time step is set to six. The GMRES solver tolerance was set to $10^{-3}$ for WD2, $10^{-4}$ for WD3, and $10^{-6}$ for TWM. With these settings, TWM required all six nonlinear iterations per time step to achieve the prescribed nonlinear solution error tolerance, WD3 converged within four-to-six nonlinear iterations, and WD2 required four or fewer nonlinear iterations for convergence. For the turbulent channel flow at $R e_{\tau}=395$ and on average, the number of GMRES iterations, added over all nonlinear iterations in a given time step, were 995 for TWM, 490 for WD3, and 352 for WD2. For the turbulent channel flow at $R e_{\tau}=950$, the average number of GMRES iterations were 1403 for TWM, 858 for WD3, and 672 
for WD2. These statistics suggest that the TWM is the "most nonlinear" of all the methods proposed, which, together with the need to average over homogeneous directions, drives up the computational costs. WD3 is a slightly more costly than WD2 to execute, however, we feel it is a small price to pay for better alignment with the law-of-the-wall.

\section{Conclusions and Future Work}

Weakly enforced no-slip wall boundary conditions were revisited in the context of LES with near-wall modeling of turbulent boundary layers. The existing weak-boundary-condition formulations, while possessing good numerical stability and accuracy properties, were found to be not completely consistent with the law-of-the-wall, because: 1 . Only the penalty term was representative of the shear stress at the wall, while the resolved molecular viscous shear stress was neglected; 2. The resolved velocity at the wall, instead of that inside the log layer, was employed to compute the wall shear stress, and the associated penalty parameter $\tau_{B}$.

In the present paper a new weakly enforced boundary-condition formulation is proposed, which addresses the above shortcomings while retaining the good numerical stability and accuracy properties of the discrete formulation necessary for robust deployment in practical applications. The new formulation is tested using 
a turbulent open-channel flow at $R e_{\tau}=395$ and 950 and compared with its predecessors, as well as with DNS. It is found that the new formulation gives closer agreement with a traditional near-wall model and DNS in the prediction of mean flow and velocity fluctuations than its earlier versions. It is important to note that no additional stabilizing mechanisms, such as averaging over homogeneous directions, were necessary to stabilize the new formulation, which is a big advantage over traditional near-wall modeling techniques.

Although the new formulation is founded on a better theoretical footing, and promising results are obtained using a challenging benchmark problem, it would be important in the future work to examine how the proposed method performs in more realistic situations involving wall-bounded turbulent flows.

\section{Acknowledgements}

The first two authors were supported by the NSF Grant No. 0846510. The last author was supported by the ARO Grant No. W911NF-14-1-0296.

\section{References}

[1] I. Akkerman, Y. Bazilevs, D. J. Benson, M. W. Farthing, and C. E. Kees. Free-surface flow and fluid-object interaction modeling with emphasis on ship hydrodynamics. Journal of Applied Mechanics, 79:010905, 2012. 
[2] I. Akkerman, Y. Bazilevs, V.M. Calo, T.J.R. Hughes, and S. Hulshoff. The role of continuity in residual-based variational multiscale modeling of turbulence. Computational Mechanics, 41:371-378, 2008.

[3] D.N. Arnold, F. Brezzi, B. Cockburn, and L.D. Marini. Unified analysis of Discontinuous Galerkin methods for elliptic problems. SIAM Journal of Numerical Analysis, 39:1749-1779, 2002.

[4] Y. Bazilevs and I. Akkerman. Large eddy simulation of turbulent TaylorCouette flow using isogeometric analysis and the residual-based variational multiscale method. Journal of Computational Physics, 229(9):3402 - 3414, 2010.

[5] Y. Bazilevs, V. M. Calo, T. J. R. Hughes, and Y. Zhang. Isogeometric fluidstructure interaction: theory, algorithms, and computations. Computational Mechanics, 43:3-37, 2008.

[6] Y. Bazilevs, V.M. Calo, J.A. Cottrel, T.J.R. Hughes, A. Reali, and G. Scovazzi. Variational multiscale residual-based turbulence modeling for large eddy simulation of incompressible flows. Computer Methods in Applied Mechanics and Engineering, 197:173-201, 2007.

[7] Y. Bazilevs, M.-C. Hsu, K. Takizawa, and T. E. Tezduyar. ALE-VMS and 
ST-VMS methods for computer modeling of wind-turbine rotor aerodynamics and fluid-structure interaction. Mathematical Models and Methods in Applied Sciences, 22(supp02):1230002, 2012.

[8] Y. Bazilevs and T.J.R. Hughes. Weak imposition of Dirichlet boundary conditions in fluid mechanics. Computers and Fluids, 36:12-26, 2007.

[9] Y. Bazilevs, C. Michler, V. M. Calo, and T. J. R. Hughes. Isogeometric variational multiscale modeling of wall-bounded turbulent flows with weakly enforced boundary conditions on unstretched meshes. Computer Methods in Applied Mechanics and Engineering, 199:780-790, 2010.

[10] Y. Bazilevs, C. Michler, V.M. Calo, and T.J.R. Hughes. Weak Dirichlet boundary conditions for wall-bounded turbulent flows. Computer Methods in Applied Mechanics and Engineering, 196:4853-4862, 2007.

[11] Y. Bazilevs, K. Takizawa, and T. E. Tezduyar. Computational FluidStructure Interaction: Methods and Applications. Wiley, 2013.

[12] J. Bredberg. On the wall boundary condition for turbulence models. Technical report, Department of Thermo and Fluid Dynamics, Chalmers University of Technology, 2000. 
[13] A.N. Brooks and T.J.R. Hughes. Streamline upwind/Petrov-Galerkin formulations for convection dominated flows with particular emphasis on the incompressible Navier-Stokes equations. Computer Methods in Applied Mechanics and Engineering, 32:199-259, 1982.

[14] J. Chung and G. M. Hulbert. A time integration algorithm for structural dynamics with improved numerical dissipation: The generalized- $\alpha$ method. Journal of Applied Mechanics, 60:371-75, 1993.

[15] J.A. Cottrell, T.J.R. Hughes, and Y. Bazilevs. Isogeometric Analysis: Toward Integration of CAD and FEA. Wiley, Chichester, 2009.

[16] J. C. del Alamo, J. Jimenez, P. Zandonade, and R. D. Moser. Scaling of the energy spectra of turbulent channels. Journal of Fluid Mechanics, 500:135$144,2004$.

[17] Juan C. del Alamo and Javier Jímenez. Spectra of the very large anisotropic scales in turbulent channels. Physics of Fluids, 15(6):L41-L44, 2003.

[18] M.-C. Hsu, I. Akkerman, and Y. Bazilevs. Wind turbine aerodynamics using ALE-VMS: Validation and the role of weakly enforced boundary conditions. Computational Mechanics, 50:499-511, 2012. 
[19] M.-C. Hsu and Y. Bazilevs. Fluid-structure interaction modeling of wind turbines: simulating the full machine. Computational Mechanics, 50:821833, 2012.

[20] T. J. R. Hughes, W. K. Liu, and T. K. Zimmermann. Lagrangian-Eulerian finite element formulation for incompressible viscous flows. Computer Methods in Applied Mechanics and Engineering, 29:329-349, 1981.

[21] T. J. R. Hughes, L. Mazzei, and K. E. Jansen. Large-eddy simulation and the variational multiscale method. Computing and Visualization in Science, $3: 47-59,2000$.

[22] T.J.R. Hughes, J.A. Cottrell, and Y. Bazilevs. Isogeometric analysis: CAD, finite elements, NURBS, exact geometry, and mesh refinement. Computer Methods in Applied Mechanics and Engineering, 194:4135-4195, 2005.

[23] T.J.R. Hughes, G. Scovazzi, and L.P. Franca. Multiscale and stabilized methods. In E. Stein, R. de Borst, and T. J. R. Hughes, editors, Encyclopedia of Computational Mechanics, Vol. 3, Computational Fluid Dynamics, chapter 2. Wiley, 2004.

[24] K. E. Jansen, C. H. Whiting, and G. M. Hulbert. A generalized- $\alpha$ method for integrating the filtered Navier-Stokes equations with a stabilized finite 
element method. Computer Methods in Applied Mechanics and Engineering, 190:305-319, 1999.

[25] C. Johnson. Numerical solution of partial differential equations by the finite element method. Cambridge University Press, Sweden, 1987.

[26] R. D. Moser, J. Kim, and N. N. Mansour. Direct numerical simulation of turbulent channel flow. Physics of Fluids, 11:943-945, 1999.

[27] J. Nitsche. Uber ein variationsprinzip zur losung von Dirichlet-problemen bei verwendung von teilraumen, die keinen randbedingungen unterworfen sind. Abh. Math. Univ. Hamburg, 36:9-15, 1971.

[28] U. Piomelli and E. Ballaras. Wall-layer models for large-eddy simulations. Annual Review of Fluid Mechanics, 34:349-374, 2002.

[29] S. B. Pope. Turbulent Flows. Cambridge University Press, Cambridge, 2000.

[30] Y. Saad and M.H. Schultz. GMRES: A generalized minimal residual algorithm for solving nonsymmetric linear systems. SIAM Journal of Scientific and Statistical Computing, 7:856-869, 1986.

[31] P. Sagaut. Large Eddy Simulation for Incompressible Flows: An Introduction. Springer, 2006. 
[32] D. B. Spalding. A single formula for the law of the wall. Journal of Applied Mechanics, 28:444-458, 1961.

[33] H. Suito, K. Takizawa, V. Q. H. Huynh, D. Sze, and T. Ueda. FSI analysis of the blood flow and geometrical characteristics in the thoracic aorta. Computational Mechanics, 54:1035-1045, 2014.

[34] K. Takizawa. Computational engineering analysis with the new-generation space-time methods. Computational Mechanics, 54:193-211, 2014.

[35] K. Takizawa, Y. Bazilevs, and T. E. Tezduyar. Space-time and ALEVMS techniques for patient-specific cardiovascular fluid-structure interaction modeling. Archives of Computational Methods in Engineering, 19:171$225,2012$.

[36] K. Takizawa, Y. Bazilevs, T. E. Tezduyar, C. C. Long, A. L. Marsden, and K. Schjodt. ST and ALE-VMS methods for patient-specific cardiovascular fluid mechanics modeling. Mathematical Models and Methods in Applied Sciences, 24:2437-2486, 2014.

[37] K. Takizawa, B. Henicke, A. Puntel, N. Kostov, and T. E. Tezduyar. Spacetime techniques for computational aerodynamics modeling of flapping wings of an actual locust. Computational Mechanics, 50:743-760, 2012. 
[38] K. Takizawa, N. Kostov, A. Puntel, B. Henicke, and T. E. Tezduyar. Spacetime computational analysis of bio-inspired flapping-wing aerodynamics of a micro aerial vehicle. Computational Mechanics, 50:761-778, 2012.

[39] K. Takizawa, D. Montes, S. McIntyre, and T. E. Tezduyar. Space-time VMS methods for modeling of incompressible flows at high Reynolds numbers. Mathematical Models and Methods in Applied Sciences, 23:223-248, 2013.

[40] K. Takizawa, K. Schjodt, A. Puntel, N. Kostov, and T. E. Tezduyar. Patientspecific computational analysis of the influence of a stent on the unsteady flow in cerebral aneurysms. Computational Mechanics, 51:1061-1073, 2013.

[41] K. Takizawa and T. E. Tezduyar. Multiscale space-time fluid-structure interaction techniques. Computational Mechanics, 48:247-267, 2011.

[42] K. Takizawa, T. E. Tezduyar, A Buscher, and S. Asada. Space-time fluid mechanics computation of heart valve models. Computational Mechanics, 54:973-986, 2014.

[43] K. Takizawa, T. E. Tezduyar, A Buscher, and S. Asada. Space-time interface-tracking with topology change (ST-TC). Computational Mechanics, 54:955-971, 2014. 
[44] K. Takizawa, T. E. Tezduyar, and N. Kostov. Sequentially-coupled spacetime FSI analysis of bio-inspired flapping-wing aerodynamics of an MAV. Computational Mechanics, 54:213-233, 2014.

[45] K. Takizawa, T. E. Tezduyar, S. McIntyre, N. Kostov, R. Kolesar, and C. Habluetzel. Space-time VMS computation of wind-turbine rotor and tower aerodynamics. Computational Mechanics, 53:1-15, 2014.

[46] A.E. Tejada-Martínez, I. Akkerman, and Y. Bazilevs. Large-eddy simulation of shallow water Langmuir turbulence using isogeometric analysis and the residual-based variational multiscale method. Journal of Applied Mechanics, 79:010909, 2012.

[47] T. E. Tezduyar, S. Mittal, S. E. Ray, and R. Shih. Incompressible flow computations with stabilized bilinear and linear equal-order-interpolation velocity-pressure elements. Computer Methods in Applied Mechanics and Engineering, 95:221-242, 1992.

[48] M.F. Wheeler. An elliptic collocation-finite element method with interior penalties. SIAM Journal of Numerical Analysis, 15:152-161, 1978. 

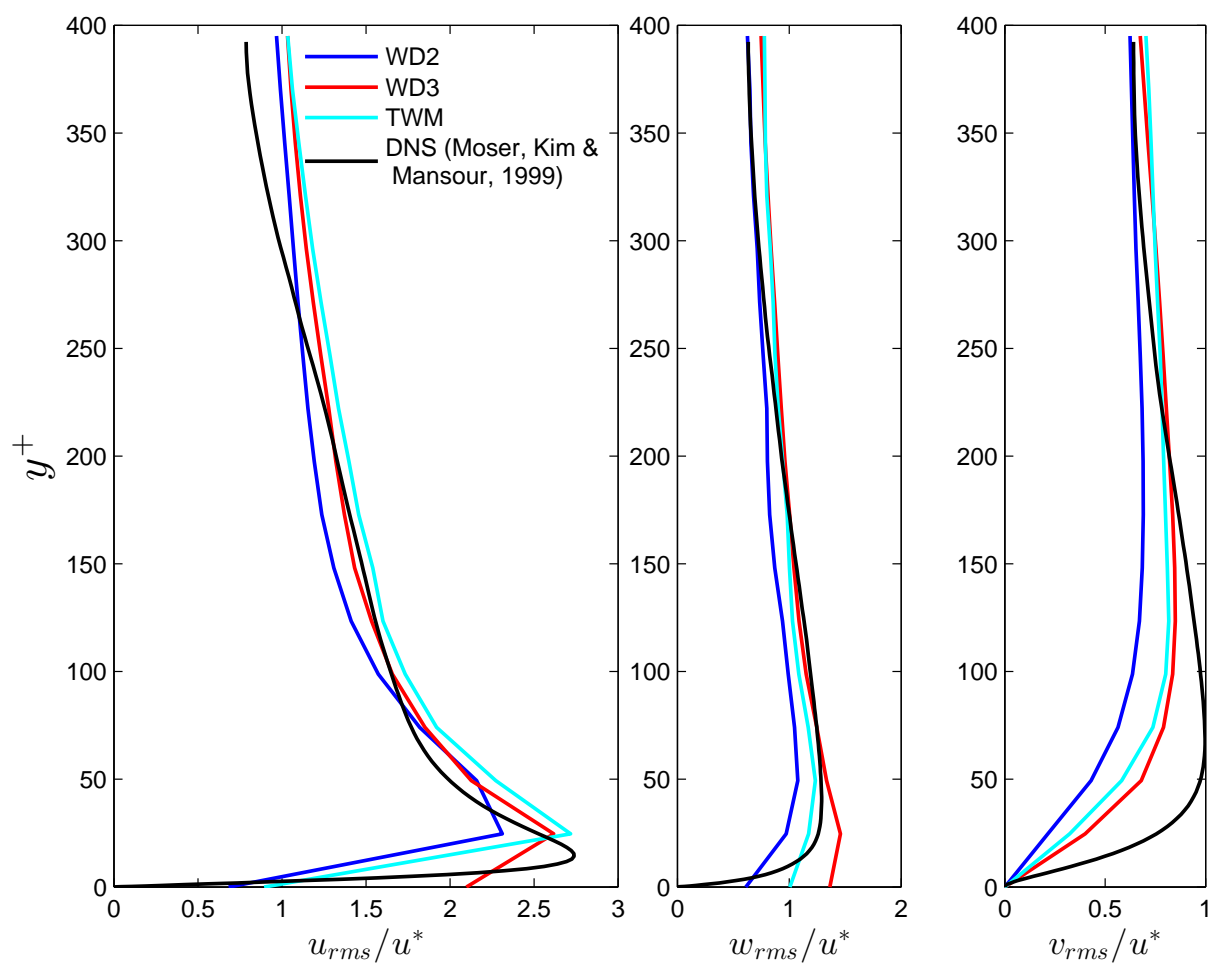

Figure 4: Root mean square (rms) of velocity in wall units from the near-wall model computations of turbulent channel flow at $R e_{\tau}=395$. DNS results are also plotted for comparison. Stream-wise velocity rms $\left(u_{r m s}\right)$ is in the left panel, span-wise velocity $\mathrm{rms}\left(w_{r m s}\right)$ is in the middle panel, and wall-normal velocity rms $\left(v_{r m s}\right)$ is in the right panel. 

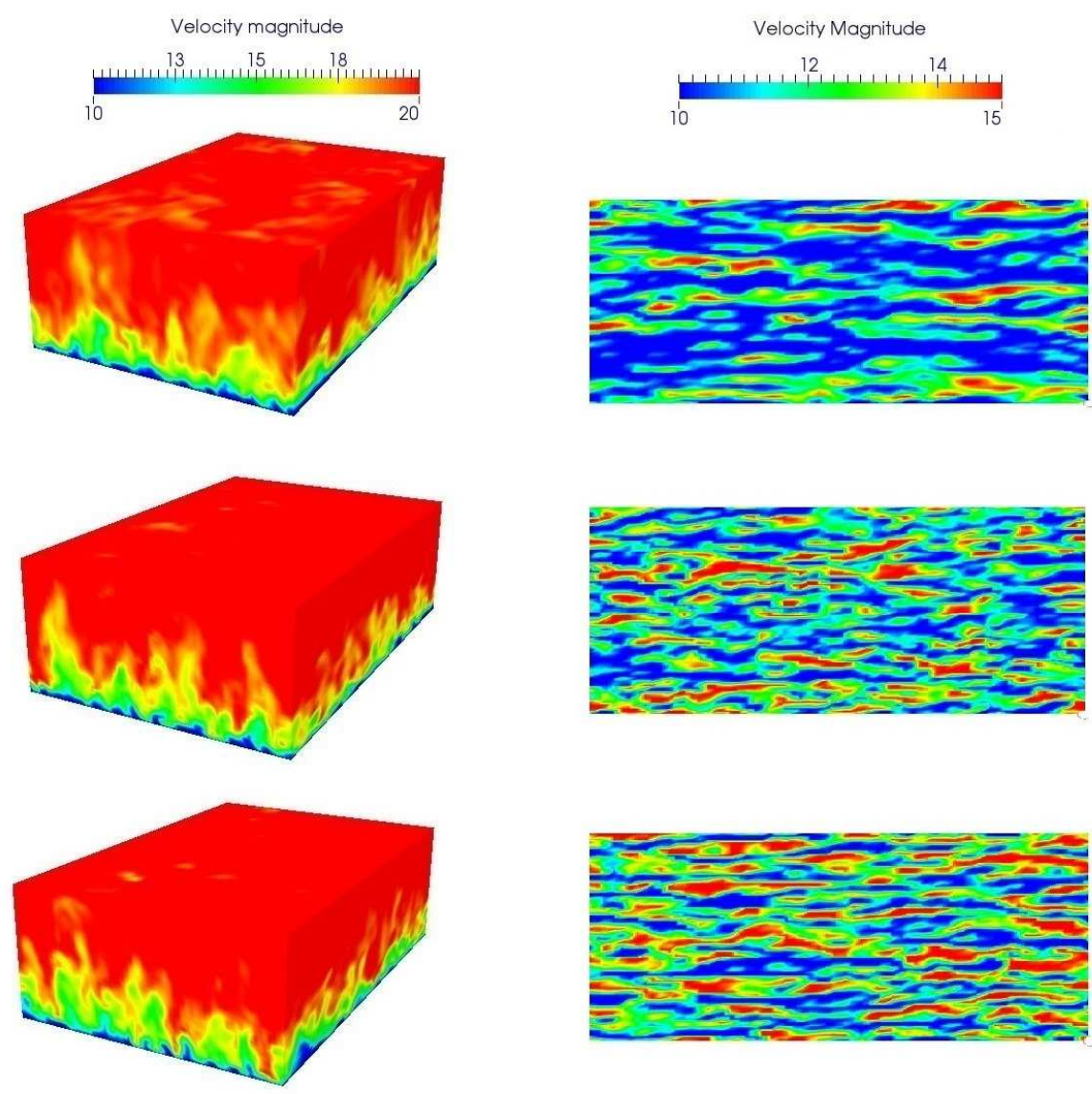

Figure 5: Instantaneous flow speed contours of turbulent channel flow at $R e_{\tau}=395$ : Full domain (left panel); Near-wall plane at $y^{+}=24.7$ (right panel). Top-to-bottom: WD2, WD3, and TWM simulation results. 


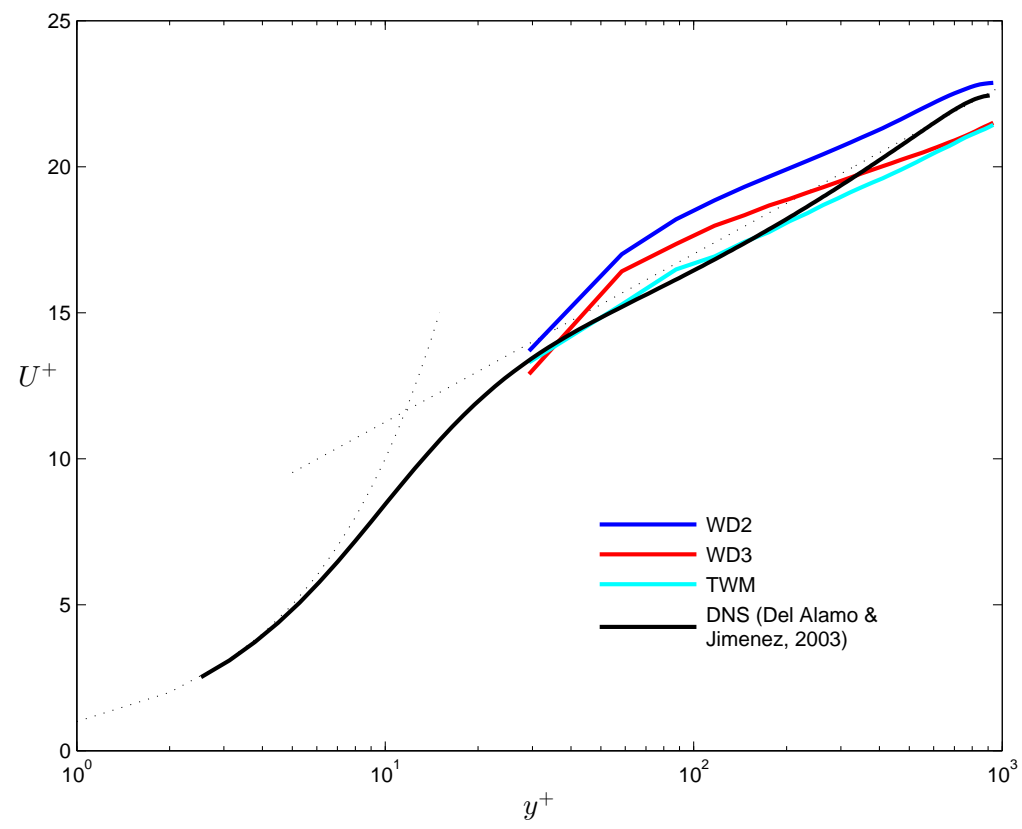

Figure 6: Mean velocity in log wall units from the near-wall model computations of turbulent channel flow at $R e_{\tau}=950$. DNS results from [16] and the theoretical $\log$ law with $\kappa=0.41$ and $B=5.5$ are also plotted for comparison. 


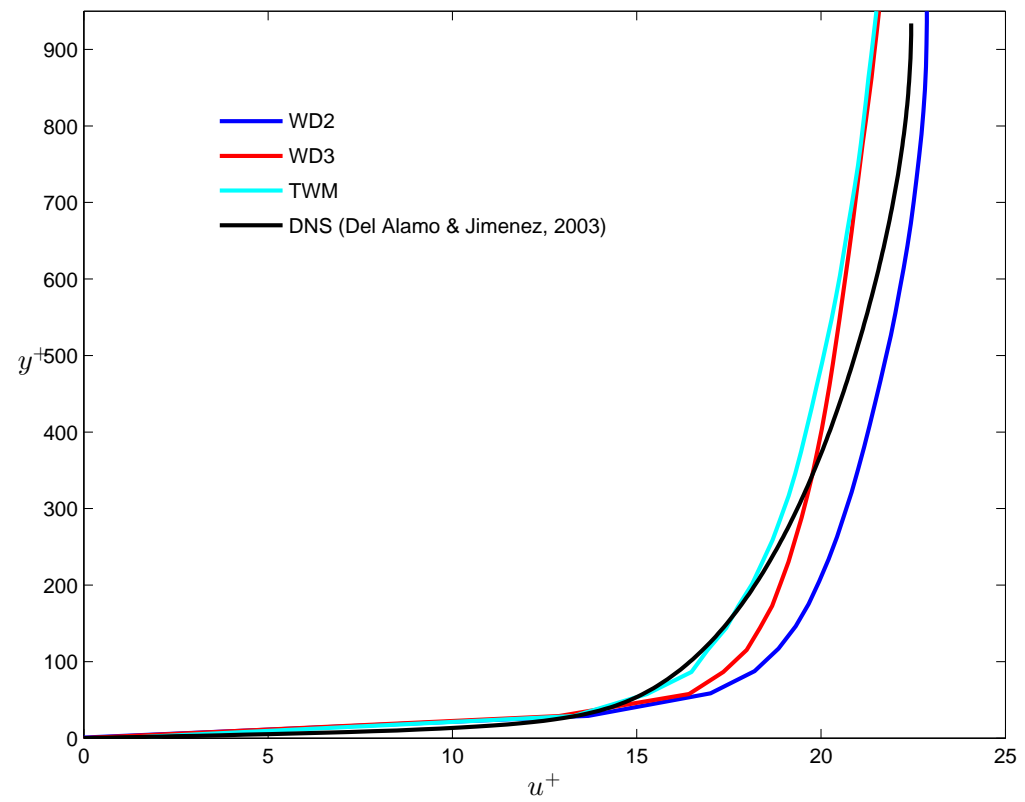

Figure 7: Mean velocity in wall units from the near-wall model computations of turbulent channel flow at $R e_{\tau}=950$. DNS results are also plotted for comparison. 

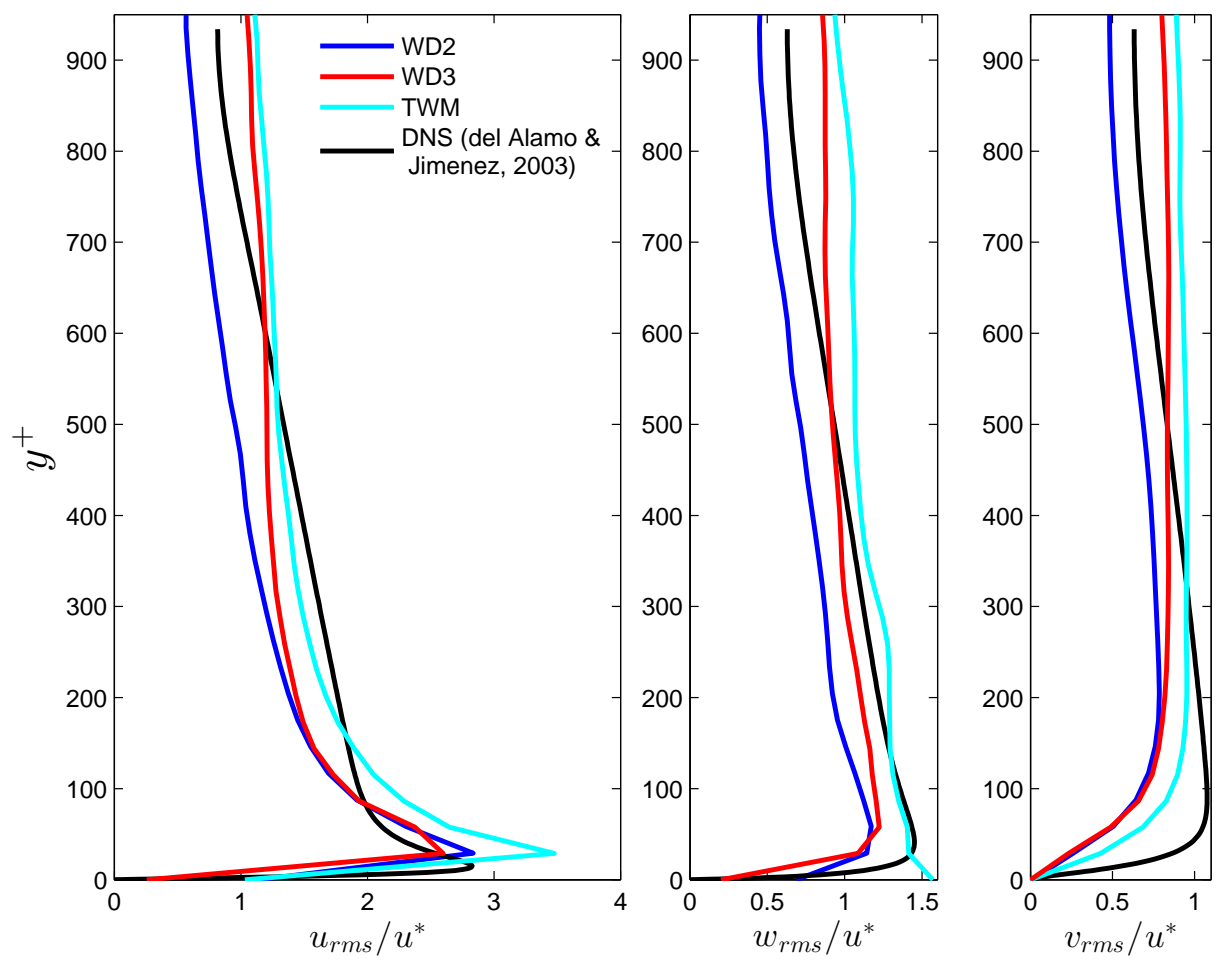

Figure 8: Root mean square (rms) of velocity in wall units from the near-wall model computations of turbulent channel flow at $R e_{\tau}=950$. DNS results are also plotted for comparison. Stream-wise velocity rms $\left(u_{r m s}\right)$ is in the left panel, span-wise velocity rms $\left(w_{r m s}\right)$ is in the middle panel, and wall-normal velocity rms $\left(v_{r m s}\right)$ is in the right panel. 

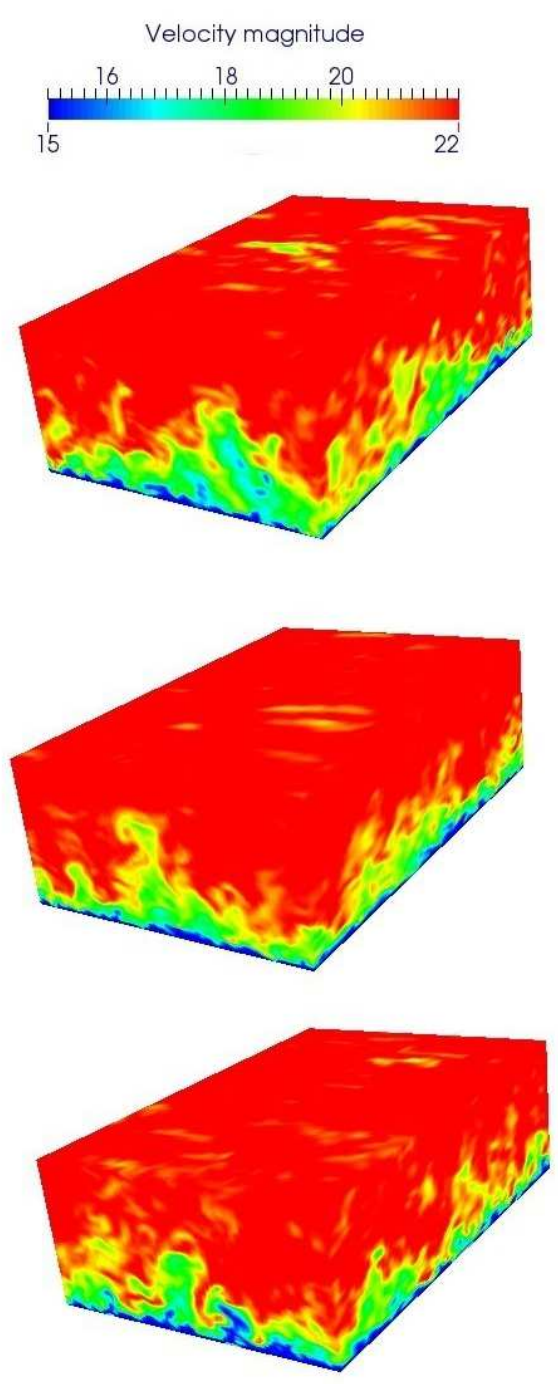
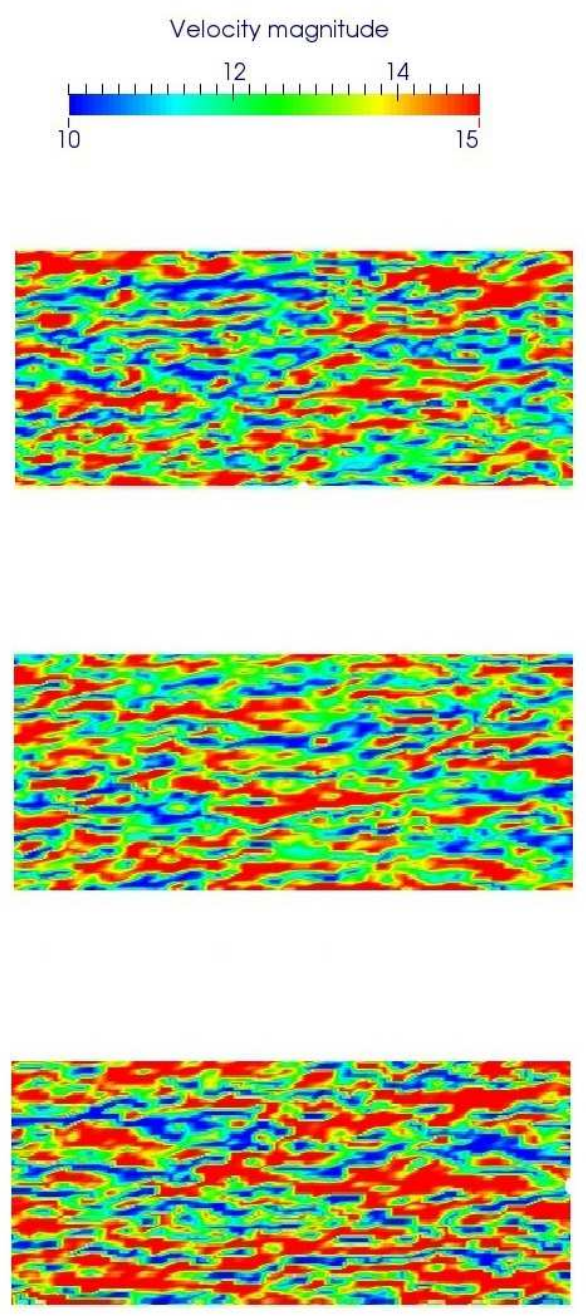

Figure 9: Instantaneous flow speed contours of turbulent channel flow at $R e_{\tau}=950$ : Full domain (left panel); Plane at $y^{+}=29.7$ (right panel). Top-to-bottom: WD2, WD3, and TWM simulation results. 